\title{
Modelo de Gestión del Conocimiento para la Integración de plataformas académicas apoyada en Aulas Virtuales
}

\author{
Víctor Hugo Medina García, PhD ${ }^{1}$, Santiago Angarita Benavides, Est ${ }^{1}$ Nicolás Hernández López, Est ${ }^{1}$ \\ ${ }^{1}$ Universidad Distrital Francisco José de Caldas, Colombia, vmedina@udistrital.edu.co, bjangarita@correo.udistrital.edu.co, \\ nihernandezl@correo.udistrital.edu.co
}

\begin{abstract}
Resumen- Las universidades disponen de diferentes plataformas en línea, que operan independientemente según la finalidad de cada una de estas. Entre estos portales web no hay una comunicación directa, para enlazar los datos automáticamente de un portal a otro.

Este estudio plantea como caso de uso la aplicación en la Universidad Distrital "Francisco José de Caldas", para vincular las bases de datos de dos de estas plataformas usadas por la universidad, las cuales son: El sistema de gestión académica (SGA) y el sistema de la Facultad de Ingeniería (UDIN).

Por tanto, se presenta un modelo de un sistema de gestión de conocimiento que fortalece y facilita a los usuarios de estas plataformas enlazar y gestionar información desde las aulas virtuales en la plataforma correspondiente a la universidad según se requiera.
\end{abstract}

El modelo propuesto contribuye a optimizar las tareas que implican asignar algún tipo de dato de un perfil en la base de datos de una de estas plataformas, de manera inmediata y automática, al perfil del mismo usuario que se encuentra en la otra plataforma.

Keywords-- Aulas virtuales, plataforma en línea, sistema de gestión académica, UDIN, vincular, bases de datos.

Abstract- Universities have different online platforms, separated according to the purpose of each one of these. There is no direct communication between these web portals, to automatically link data from one portal to another.

This study proposes as a use case the application in the Universidad Distrital "Francisco José de Caldas", to link the databases of two of these platforms used by the university, which are: The academic management system (SGA) and UDIN Faculty of Engineering (UDIN).

Therefore, below is a model of a knowledge management system that allows users of these platforms to link information from virtual classrooms on the UDIN platform to the EMS as required.

The proposed model will contribute to optimizing the tasks that involve assigning some type of data from a profile in the database of one of these platforms, immediately and automatically, to the profile of the same user who is on the other platform.

Keywords-- Virtual classrooms, online platform, academic management system, UDIN, link, databases.

\section{INTRODUCCIÓN}

Las plataformas en línea se han convertido en una herramienta fundamental y de gran utilidad para gestionar el

Digital Object Identifier (DOI):

http://dx.doi.org/10.18687/LACCEI2021.1.1.256

ISBN: 978-958-52071-8-9 ISSN: 2414-6390 conocimiento en la Universidad Distrital Francisco José de Caldas en Bogotá - Colombia, esta institución cuenta con diversas plataformas, cada una de ellas con diferentes enfoques para dar soporte a diversos tipos de información.

Entre estas se encuentran el sistema de gestión académica (SGA), en donde se puede encontrar información acerca de los perfiles de estudiantes y profesores, para asignar horarios de los diferentes espacios académicos y asignar sus respectivas notas entre otras funciones; otra plataforma con la que cuenta la universidad es el sistema de Facultad de Ingeniería (UDIN) en donde se puede encontrar información relacionada a la institución y las diferentes sub-ramas que competen a la Facultad de Ingeniería, como lo son las aulas virtuales de los diferentes espacios académicos de esta facultad, permitiendo a los profesores y estudiantes tener un espacio en la web para desarrollar diversas actividades académicas que complementen el trabajo hecho en clase.

Debido a que no hay un sistema que relacione la información de una plataforma a otra, que permita, por ejemplo, asociar la nota de una actividad realizada por un estudiante en determinada aula virtual de UDIN a su sábana o registro de notas en su respectivo perfil del SGA de manera automática se plantea un modelo que permita vincular la base de datos del SGA con las aulas virtuales de la plataforma de UDIN.

\section{CONCEPTUALIZACIÓN}

A la hora de confrontar uno o más conceptos es necesario definir, clasificar y enfatizar los aspectos importantes de cada uno de ellos para establecer sus relaciones, y en este trabajo se definen aulas virtuales y sus generalidades.

\section{A. Aulas virtuales}

El aula virtual es un sistema en la WWW en la cual los educadores y educandos se encuentran para realizar actividades involucradas en el proceso de aprendizaje, es decir que deben permitir interactividad, comunicación, aplicación de los conocimientos, evaluación y manejo de la clase [1]. Algunas son sistemas cerrados en los que el usuario se limitar a las opciones que fueron pensadas por los creadores del espacio virtual, para desarrollar su curso. Otras se extienden a lo largo y a lo ancho de la red usando el hipertexto como su mejor aliado para que los alumnos no dejen de visitar o conocer otros recursos en la red relacionados a la clase [2].

$19^{\text {th }}$ LACCEI International Multi-Conference for Engineering, Education, and Technology: "Prospective and trends in technology and skills for sustainable social development" "Leveraging emerging technologies to construct the future", Buenos Aires -Argentina, July 21-23, 2021. 
El apoyo tecnológico de las plataformas está direccionado a tres tipos de perfiles:

- Administrador del sitio

- El docente

- Los estudiantes

Cada uno de ellos maneja un nombre de usuario con su respectiva contraseña y tiene un lugar virtual además de trabajo y funciones específicas.

Para que cada perfil cumpla con los objetivos propuestos en el proceso de enseñanza aprendizaje la plataforma debe ofrecer las siguientes características:

- Herramientas de gestión de contenidos: archivos con contenidos conceptuales

- Herramientas de comunicación y colaboración: espacios para comunicarse y ayuda recíproca

- Herramientas de seguimiento y evaluación: sirve hacer seguimiento evaluativo por dimensiones

- Herramientas de administración y asignación de permisos

- Herramientas complementarias: bibliografía de páginas sirvan para retroalimentar los temas.

Las plataformas educativas se utilizan en la educación presencial, semipresencial, a distancia, o por personas que crean espacios con intereses educativos [4].

\section{B. Sistemas de Gestión del Conocimiento}

Para lograr generar un concepto de sistema de gestión de conocimiento, es importante definir cada uno de los componentes que lo conforman:

a. Conocimiento: Es como aquella información almacenada en una entidad y que puede ser utilizada por la inteligencia de acuerdo a ciertos objetivos. Se puede dividir el conocimiento en dos grupos [5]:

- Natural que pertenece a los organismos vivos con sistema nervioso.

- Artificial, que poseen aquellos mecanismos que simulan o reproducen parcialmente al sistema natural.

- En el caso del hombre, el conocimiento es producto de procesos mentales que parten de la percepción, el razonamiento o la intuición. En ese sentido, uno de los conceptos fundamentales para el conocimiento es la capacidad de relación o asociación entre estos.

$b$. Componentes del conocimiento: El conocimiento reside en el complejo sistema de procesos que da como resultado, la materialización de los bienes o servicios. Existen dos soportes básicos del conocimiento [6]:

- Los recursos humanos que intervienen en los procesos de producción o de soporte organizacional (formación, capacidades, cualidades personales, entre otras).

- La información manejada en dichos procesos, que capacita a estas personas a incrementar su formación o habilidades para el desarrollo de sus tareas.

c. Gestión del conocimiento: Es un proceso que apoya a las organizaciones para encontrar la información relevante, seleccionar, organizar y comunicarla a todo el personal activo; este ciclo es necesario para acciones tales como la resolución de problemas, dinámica el aprendizaje y la toma de decisiones.

La gestión del conocimiento puede mejorar el desempeño de la organización en vías de lograr una organización inteligente, pero no es suficiente por sí sola; puesto que este involucra la planeación de estrategias y el establecimiento de políticas y además la colaboración de todo el personal de la organización un alto sentido de compromiso para ejecutar su trabajo y la aceptación del proceso [7].

d. Componentes de la gestión del conocimiento: Esta claro que las personas están en el centro de la gestión del conocimiento. Pero a nosotros nos interesan sobre todo sus habilidades y su información. Estos dos componentes son básicos a la hora de tener en cuenta el proceso de Gestión del conocimiento en una organización.

La información puede ser recogida tratada y almacenada por los sistemas de información de la empresa facilitando la creación de un cuadro de mando del entorno y un cuadro de mando integral para la dirección. Si estos sistemas están bien diseñados obtendremos información periódica y sistemática de lo que ocurre tanto dentro como fuera de la organización y podremos tomar decisiones con rapidez

Las habilidades son en cambio más complicadas de trasmitir ya que implica adquirir nuevo conocimiento para el que las recibe lo cual supone más tiempo [8].

e. Concepto de Sistema de Gestión del Conocimiento: Un sistema de gestión de conocimiento es conjunto de herramientas, técnicas y métodos como lo son la calidad del recurso humano, la capacidad de gestionar la información y la habilidad del modelo organizativo para implementar e integrar las herramientas, técnicas y métodos adecuados; plasmados en las organizaciones públicas y privadas. La principal característica funcional del sistema de gestión del conocimiento es hacer coincidir las necesidades concretas de información de las distintas personas y equipos de trabajo con la disponibilidad efectiva de dicha información.

De esta concepción acerca del conocimiento y la información manejada han surgido nuevas áreas de trabajo vinculadas a la creación de nuevos modelos organizacionales. Sobre la base de lo anterior, se puede señalar que una organización es una suprarred de recursos de conocimiento compuesta por una red de recursos humanos, la red de recursos de información y la red de recursos informáticos y telemáticos.

Por lo tanto, se resume como una plataforma de TIC que soporta los procesos de gestionar el conocimiento en la organización [9].

\section{ANÁLISIS DE LOS SISTEMAS DE GESTIÓN ACADÉMICO}

\section{A. Modelo del SGA}


Sistema de Administración de Información Académico, basado en tecnologías Web, orientado a servicios sobre plataforma de software libre, escalable, modular, ajustado a las necesidades de la Universidad que provee servicios web académicos y cuenta con mecanismos que garantizan la confidencialidad, integridad, disponibilidad de la información, así como la acertada y confiable autenticación de los usuarios, lo que incluye otros sistemas.

El objetivo es gestionar los Sistemas de Información y las Telecomunicaciones para asegurar el acceso, adquisición, disponibilidad, confiabilidad, confidencialidad y seguridad de los activos de información a través de la infraestructura y las soluciones Informáticas en el marco de la normatividad vigente aplicable, como apoyo a los Procesos Misionales de la Universidad para satisfacer las necesidades de la comunidad universitaria [10]. El sistema de gestión académica está compuesto por los siguientes portales:
- Portal Docente y Administrativos: Si el usuario que tiene vinculación con la Universidad como Docente, Funcionario o Contratista puede ingresar a este portal con su número de identificación y contraseña establecida.

El perfil de Docente, permite la vinculación de asignaturas, grupos de estudiantes y horarios respectivos, además de suministrar las calificaciones correspondientes a los perfiles de estudiantes en el Portal Estudiantes. Como función evaluativa se permite la evaluación de estos perfiles por parte de los perfiles de estudiantes, para evaluar su desempeño, lo que se conoce como la evaluación docente.

El perfil Administrativo, permite controlar el sistema mediante un ciclo PHVA que implica 4 pasos: planear, hacer, verificar y actuar (Fig. 1).
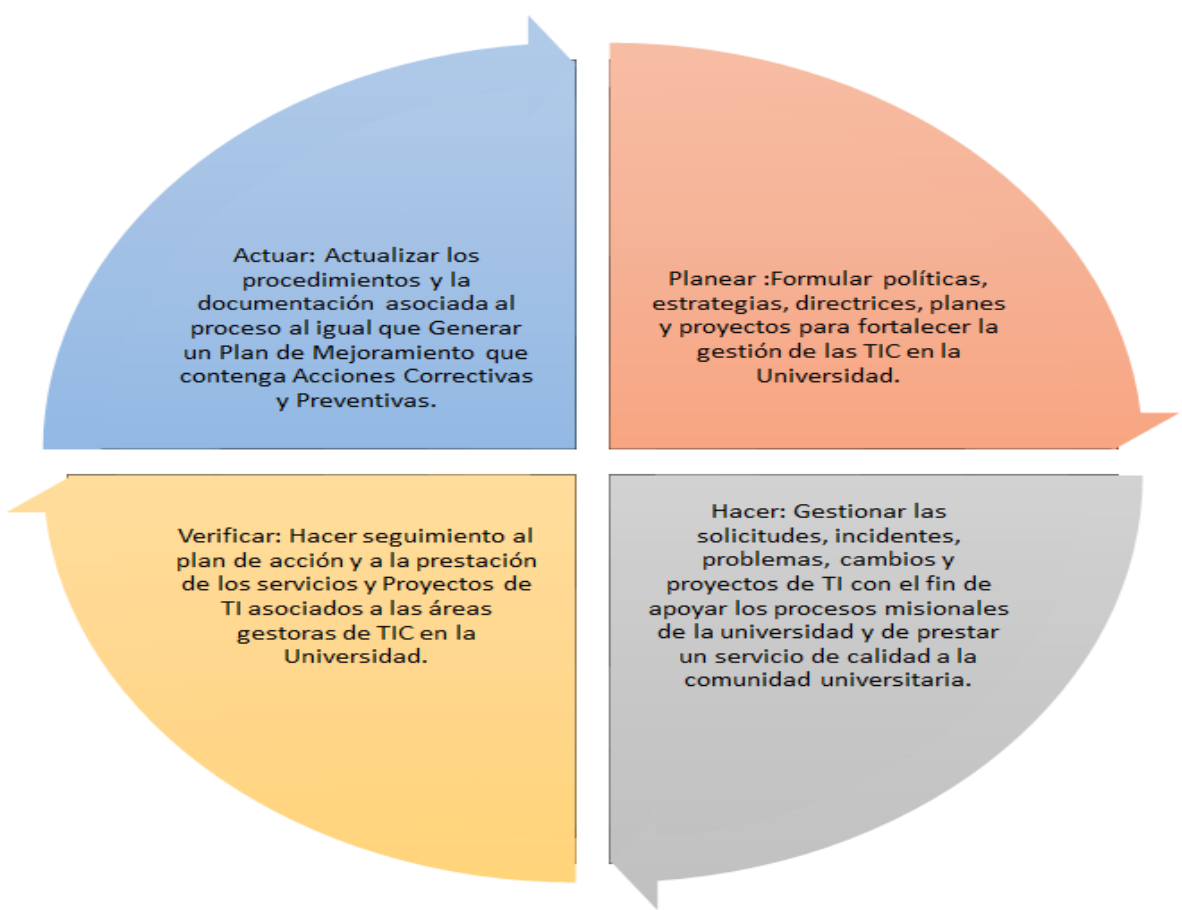

Fig. 1. Ciclo PHVA de la gestión de los sistemas de información y las telecomunicaciones en la Universidad Distrital Francisco José de Caldas. Fuente: Autores [10]

- Portal Estudiantes: Si es usuario es egresado o estudiante de la Universidad permite realizar:

- Un perfil personalizado con la información pertinente a la comunidad Universitaria.

- Adicionar, cancelar y visualizar los horarios de las asignaturas que puede cursar el respectivo usuario.

- Ser calificado por los docentes y solicitar diferentes certificados.

- Contactar y evaluar docentes.
- Acceso a diferentes servicios para el pago de matrículas, inscripción a grado, visualizar estamentos y normativas, solicitar derechos pecuniarios, visualizar plan de estudio e historia académica respectiva al perfil.

- Acceder a bases de datos de alianzas de la Universidad

- Utilizar software licenciado en alianza con la Universidad.

- Modelo de operación del SGA: El modelo de operación del sistema de Gestión Académico se presenta en la siguiente Fig. 2. 


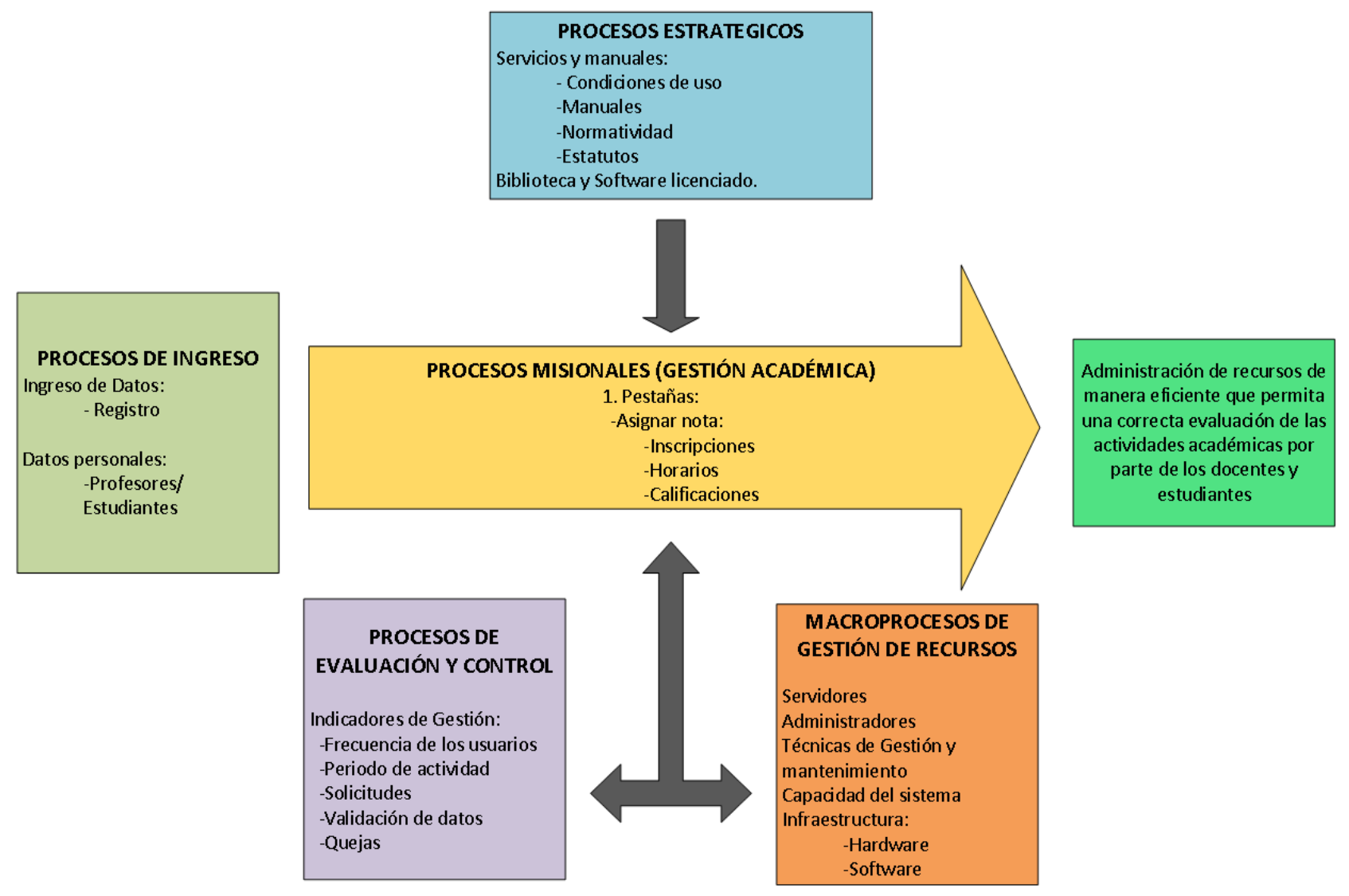

Fig. 2. Modelo de operación del SGA. Fuente Autores.

- Proceso de Ingreso: Identificación y registro según el tipo de tipo de perfil en base a la vinculación de este usuario con la Universidad.

- Procesos misionales de la Gestión Académica: Enmarca los procesos esenciales de la institución que están directamente relacionadas con la misión institucional y la satisfacción de las partes interesadas.

- Proceso Estratégico: Enmarca los procesos a través de los cuales la institución genera los lineamientos, políticas y estrategias para el desarrollo y direccionamiento de los demás procesos.

- Proceso Gestión de Recursos: Enmarca los procesos necesarios para gestionar los recursos institucionales que soportan el desarrollo de los demás procesos.

- Proceso de Evaluación y Control: Enmarca los procesos a través de los cuales se evalúa y controla el desarrollo de los demás procesos.

\section{B. Modelo de las aulas virtuales en la plataforma UDIN}

Las aulas virtuales permiten a las IES (Instituciones de Educación Superior), crear y gestionar el conocimiento dentro de la comunidad académica. Dichas aulas tienen como propósito facilitar el paso de conocimiento tácito a explícito entre alumnos y profesionales. Asimismo, en este tipo de escenarios educativos se evidencia una retroalimentación de la información, en donde todo proceso de transformación de la información en conocimiento (explícito) es guardado en un repositorio o en alguna base de datos, la cual puede verse como un proceso de transformar el conocimiento en capital intelectual, de esta forma podemos asociar este conocimiento como: "un conocimiento explícito que al fluir no envejece" [11].

Para el caso de la Universidad Distrital, la facultad de ingeniería cuenta con herramientas capaces de facilitar este conocimiento y su retroalimentación (UDIN, Google Meet, Moodle). Visto desde una perspectiva enfocada en el elearning encontramos que Rosenberg [12] define el concepto de e-learning como: "el uso de una serie amplia de tecnologías de Internet para aumentar el conocimiento y el rendimiento". Siguiendo como objetivo el crear una cultura basada en compartir el aprendizaje.

Siendo visto el e-learning como el uso adecuado de plataformas tecnológicas para la creación y socialización del conocimiento, se puede asociar la plataforma de UDIN como una herramienta tecnológica e innovadora que permite crear una cultura basada en transformar y generar nuevos saberes, los cuales serán valorados por profesionales capacitados en el área del saber (profesores). El modelo de conversión del 
conocimiento de las aulas virtuales de la universidad Distrital, se puede modelar empleando el modelo de [13], como se expone a continuación:

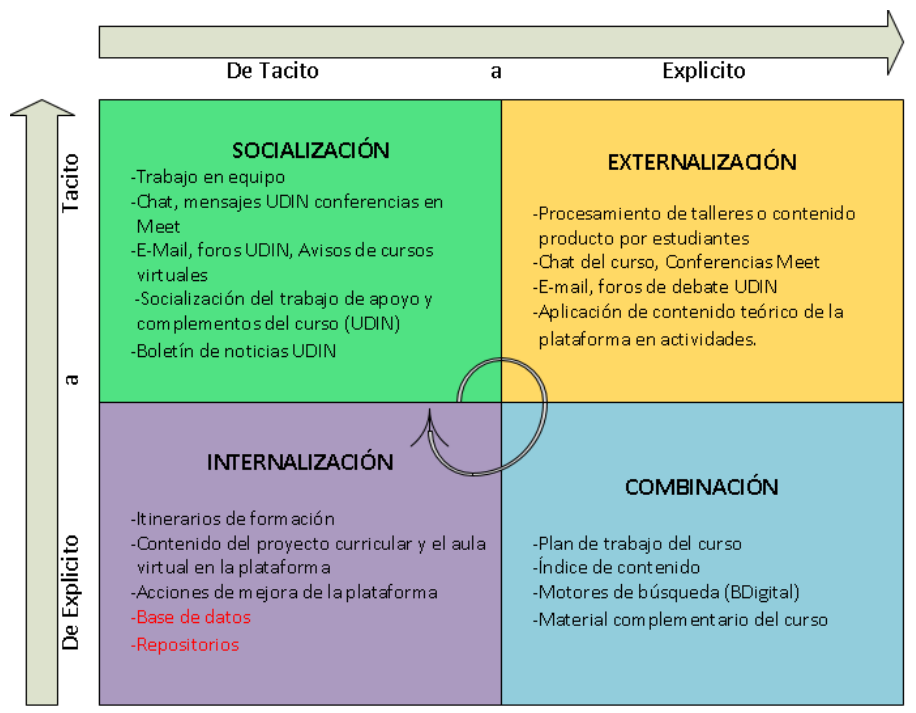

Fig. 3. Modelo de conversión del conocimiento basado en el modelo de Nonaka y Takeuchi. Fuente: Autores.
Como se puede apreciar en la figura 4, las aulas virtuales de UDIN facilitan el proceso de Socializar el conocimiento tácito mediante herramientas propias de la plataforma (Chat, mensajes, foros, boletines). Asimismo, los procesos de externalización y combinación se desarrollan empleando talleres o material de apoyo de los docentes a los estudiantes y mediante los foros de debate en lo que respecta a el primer proceso. En cambio, para la combinación se emplean los contenidos de los cursos virtuales junto con los motores de búsqueda que brinda la universidad (BDigital).

Al plantear el modelo de gestión del conocimiento para la plataforma UDIN queremos hacer un énfasis en que actualmente el proceso de internalización no se lleva a cabo correctamente, en el sentido de que los proyectos y trabajos presentados por los estudiantes son evaluados por los docentes, pero no existe un repositorio de estos ni una base de datos que permita la internalización de los mismos a la comunidad académica.

Para realizar el modelo de gestión del conocimiento de la plataforma UDIN se emplea el modelo de cuadro de mando integral, posterior a esto se aplica la mejora en la retroalimentación del conocimiento explícito generado por la comunidad.

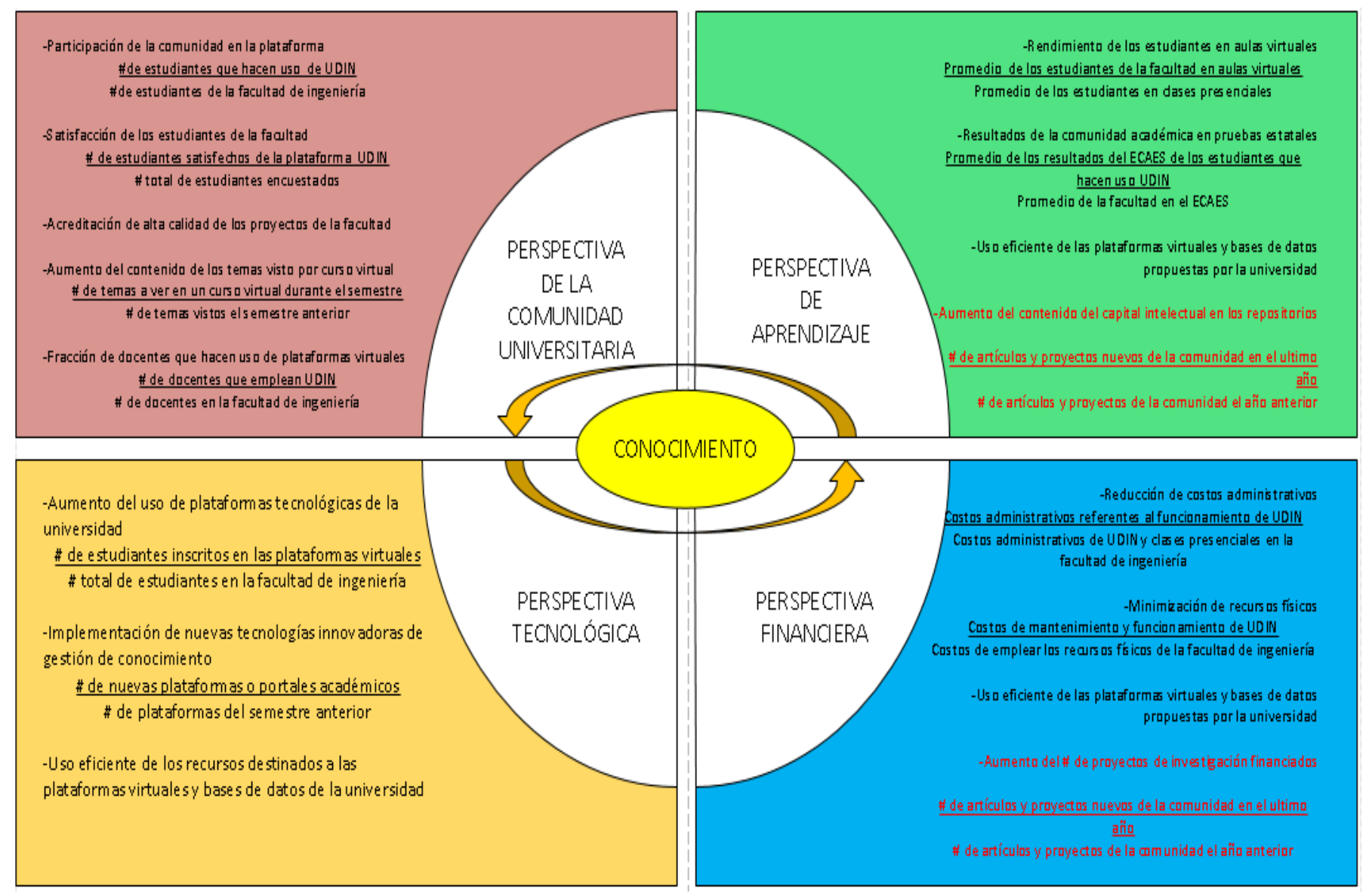

Fig. 4. Modelo de gestión del conocimiento propuesto de UDIN basado en el cuadro de mando integral de Kaplan y Norton. Fuente: Autores.

19 ${ }^{\text {th }}$ LACCEI International Multi-Conference for Engineering, Education, and Technology: "Prospective and trends in technology and skills for sustainable social development" "Leveraging emerging technologies to construct the future", Buenos Aires -Argentina, July 21-23, 2021. 
Como se venía planteando la problemática de una falta de repositorios o bases de datos para la retroalimentación del modelo, se dejan resaltados en rojo los indicadores que facilitan una métrica entre cada perspectiva del modelo en lo que respecta a el conocimiento explícito generado. Como se puede apreciar en la figura 4, no existe un vínculo directo de la plataforma con el sistema de gestión académica, lo cual genera una actividad repetitiva en las funciones de los docentes que deben evaluar los trabajos propuestos en la plataforma UDIN. A continuación, se explica cada una de las perspectivas del modelo de gestión de conocimiento para la plataforma.

- Perspectiva tecnológica: La implementación de las TIC dentro del proceso de aprendizaje nos permite explorar nuevos campos de aplicación de la pedagogía en el desarrollo profesional de los estudiantes universitarios. Dentro de esta perspectiva los medios que proporciona la universidad para facilitar el proceso de aprendizaje a larga distancia de las instalaciones de la misma deben ser usados de forma eficiente por todas las partes interesadas (administrativos, docentes y estudiantes). De esta forma la expansión y creación de plataformas innovadoras de aprendizaje lograrán disminuir costos administrativos de hacer uso de las sesiones presenciales. Así mismo las nuevas plataformas de gestión de conocimiento brindan nuevas alternativas para socializar el conocimiento explícito que se crea en dicho proceso.

- Perspectiva de aprendizaje: Al momento de proporcionar las plataformas para las aulas virtuales se debe establecer un alcance del proyecto, en el cual se tenga pensado si estas plataformas sirven como complemento a las actividades académicas presenciales o se pueden emplear como un medio de clases a distancia. De ser así, las relaciones existentes en la cantidad de usuarios en las clases virtuales deben ser valoradas en términos de resultados académicos obtenidos, los cuales se pueden valorar con exámenes estatales o el capital intelectual generado de los estudiantes en repositorios y bases de datos. Se debe buscar que las plataformas virtuales cuenten con los recursos complementarios suficientes (Google Meet, Moodle, Classroom, etc) para generar un proceso de transformación del conocimiento implícito al conocimiento explícito.

- Perspectiva financiera: Las plataformas empleadas para los cursos virtuales de la facultad permiten ahorrar recursos físicos, dado que algunos cursos se pueden desarrollar a distancia de la universidad. Dicha disminución de los costos físicos se puede ver reflejados en los costos administrativos involucrados en el desarrollo de las clases presenciales dentro del campus universitario. De esta forma la relación financiera con el proceso de aprendizaje se ve reflejada en el número de proyectos de investigación que se pueden generar en la plataforma, es por esto que el repositorio de las aulas virtuales debe ser tomado a consideración al momento de diseñarlas.

- Perspectiva de la comunidad universitaria: La comunidad universitaria y la sociedad se enfrentan día a día a un cambio cultural guiado por el avance de las tecnologías y la cibernética. Las plataformas o bases de datos empleadas para facilitar el proceso de aprendizaje deben ser valoradas por docentes y estudiantes, la métrica de estos medios permite también evaluar una acción de mejora ante el cambio de paradigma que debe afrontar la universidad en cuanto a uso de las tecnologías se refiere, dado que el conocimiento debe ser renovado y la forma de enseñarlo también.

\section{MODELO DE IMPLEMENTACIÓN DE UN SISTEMA DE GESTIÓN DEL CONOCIMIENTO ENTRE UDIN Y EL SGA}

Después de haber realizado un análisis conceptual de las aulas virtuales y de haber modelado la gestión del conocimiento en la plataforma UDIN y el Sistema de gestión académica (SGA), se procederá a realizar un modelo de gestión de conocimiento que permita la interacción entre ambas plataformas. El objeto de este modelo de gestión es realizar una administración de recursos de manera eficiente que permita una correcta evaluación de las actividades académicas por parte de docentes y estudiantes.

Asimismo, se plantea una mejora en el modelo pensando en la retroalimentación de los resultados obtenidos en el proceso de aprendizaje. Empleando un nodo adicional a los 2 modelos previamente expuestos, que permita la retroalimentación del sistema y la socialización del conocimiento explícito generado en las aulas virtuales por la comunidad académica.

El modelo propuesto para las aulas virtuales se presenta en la siguiente Fig. 5. 


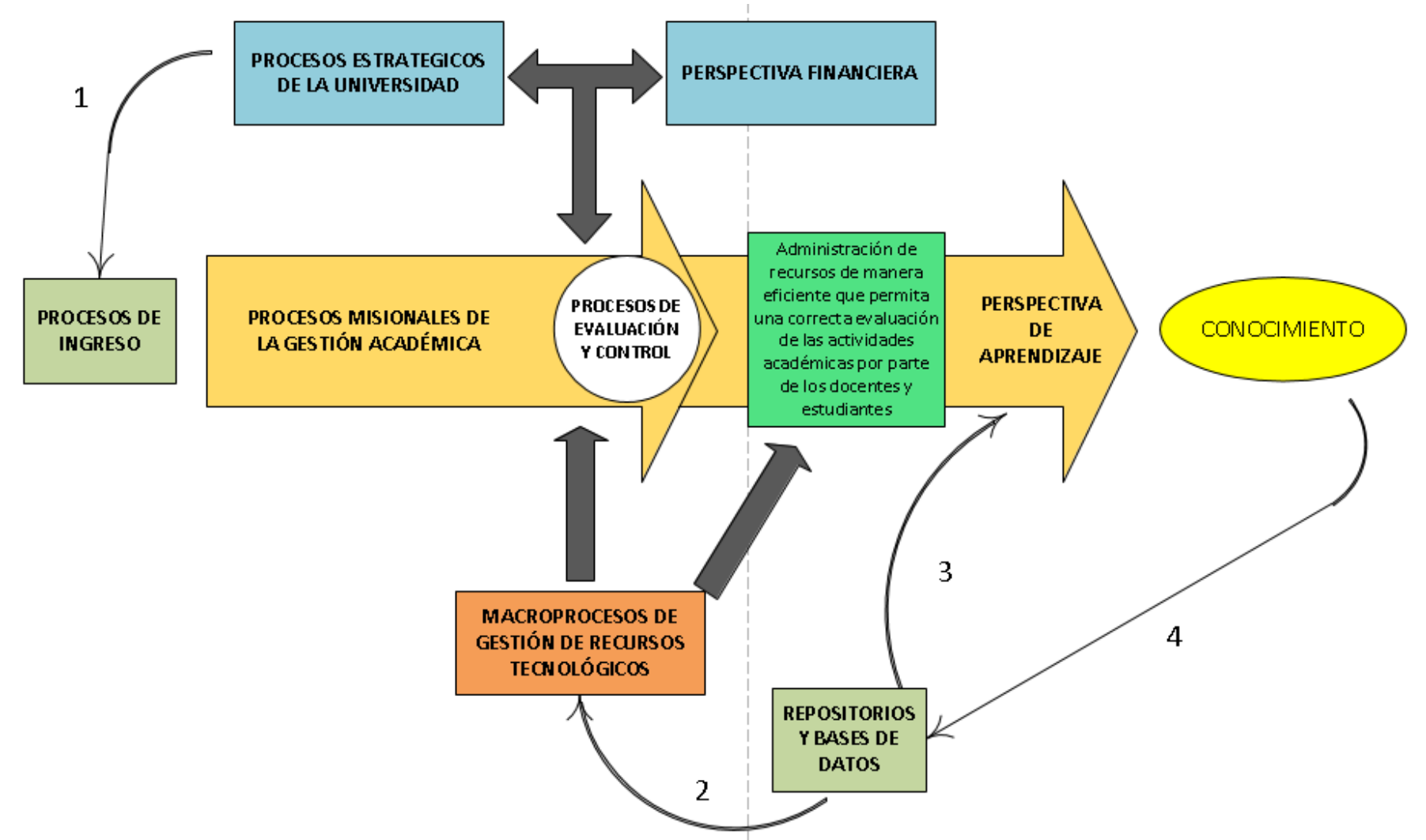

Fig. 5. Modelo de gestión del conocimiento propuesto del SGA y UDIN. Fuente: Autores.

El modelo representado en la figura 5 esquematiza una propuesta para la vinculación entre las bases de datos de las aulas virtuales de la plataforma UDIN en concordancia con los perfiles de los respectivos usuarios en el SGA, utilizando como eje de unión y propósito de este modelo la integración del conocimiento por parte de las dos plataformas por medio de los siguientes procesos:

Procesos de ingreso: Se trabajará bajo los tres perfiles base, previamente establecidos en las plataformas del SGA y UDIN (Docentes; Administradores y Estudiantes) de forma tal que los usuarios tengan una fácil adaptación a este modelo planteado. Se planteó una vinculación entre el perfil del usuario de la plataforma del SGA con su perfil correspondiente en las aulas virtuales de la plataforma UDIN en caso de docentes y estudiantes. Para los Administradores se le otorgaran permisos que permitan realizar configuraciones entre las bases de datos de ambas plataformas.

Procesos de evaluación y control: Siendo parte fundamental para la integración de estas dos plataformas se planteó generar un enlace entre las calificaciones que se le hayan asignado a un determinado usuario de una plataforma a partir de un solo perfil y de manera automática y no bifurcada como se encuentra actualmente cada sistema.

Procesos Estratégico: Enmarca los procesos a través de los cuales la institución genera los lineamientos, políticas y estrategias para la gestión, el desarrollo y direccionamiento de los demás procesos en el sistema, de forma tal que en ambos sistemas se establezcan los parámetros necesarios para que los usuarios de ambas plataformas sean vinculados de manera confiables, transitoria, y efectiva con su respectivo perfil en ambas plataformas.

Perspectiva financiera: Para la ejecución de este modelo se debe plantear una reestructuración de las plataformas de manera óptima, teniendo en cuenta los recursos que ambas requieren por separado, y un posterior análisis de costos a un plazo medio, donde se integren cargos de administradores, técnicos, contratistas y/o infraestructura que sea homologable y de fácil adaptación de un sistema a otro.

Macroprocesos de gestión de recursos tecnológicos: Se debe realizar una reestructuración de la infraestructura utilizada por cada plataforma (Servidores, Oficinas; TIC); con el propósito de fusionarlas y que sean recicladas para la ejecución del modelo planteado y de esta forma reducir el costo por el cambio de los dos modelos actuales utilizados a el modelo propuesto en la figura 5.

Procesos misionales de la Gestión académica: Enmarca los procesos esenciales de la institución que están directamente relacionadas con la misión institucional y la satisfacción de las partes interesadas. Por lo tanto, en el modelo propuesto se plantea que las diferentes actividades ejecutadas en las aulas virtuales por parte de los docentes y estudiantes se vean reflejadas de forma automática mediante arquitecturas de

$19^{\text {th }}$ LACCEI International Multi-Conference for Engineering, Education, and Technology: "Prospective and trends in technology and skills for sustainable 
llamamiento de datos en la pestaña de calificaciones, como primer objetivo de integración entre plataformas.

Perspectiva de aprendizaje: Este proceso continuará rigiéndose bajo el mismo concepto misional de la Universidad, $\mathrm{y}$ en vez de ser modificado se busca que se vea beneficiado debido a un uso más accesible y "amigable” para los diferentes usuarios de la plataforma de tal manera que el conocimiento generado circule por todo el sistema.

Dentro de las relaciones expuestas en el modelo, se procede a explicar las más relevantes:

(2) El proceso de ingreso de cualquier estudiante a la Universidad Distrital se ve restringido a medida que el candidato a ingresar cumple o no cumple con los requerimientos establecidos en las políticas dictaminadas por la institución. Encontramos indicadores como:

\section{Candidatos admitidos en el proceso de selección Candidatos presentados durante el proceso}

Nro estudiantes que abandonan la universidad en el semestre

Número de estudiantes admitidos en el actual semestre

(3) El repositorio y la base de datos requieren de una infraestructura que permita su funcionamiento y el adecuado mantenimiento de la misma. Como parte de la mejora continua del sistema, se requiere una retroalimentación con indicadores que evalúen si el funcionamiento del sistema es el apropiado, dichos indicadores pueden ser:

Tiempo total fuera de servicio y reparación

Tiempo total de operación del sistema

\# de fallas presentadas en el repositorio

Tiempo total de operación del sistema

(3 y 4) La importancia del uso de las aulas virtuales es el flujo o la "renovación" del conocimiento explícito creado durante el proceso de aprendizaje. Dicha retroalimentación permite superar los objetivos planteados y proponer acciones de mejora continua al sistema de aprendizaje. Los indicadores que destacan son:

\# artículos/proyectos de inv. publicados en semestre actual \# artículos/proyectos de inv. publicados en semestre anterior

\# artículos/proy. de inv. publicados en el semestre actual

Meta de artículos/proy. presentados en el semestre actual

\section{CONCLUSIONES}

El modelo que se plantea en el artículo integra las aulas virtuales (recursos del e-learning) y el sistema de gestión académica a manera de propuesta, con el fin de realizar un proceso de valuación eficiente por parte de los docentes, reduciendo tiempos en repetir actividades de gestión. Asimismo, la propuesta de mejora integra una alternativa para socializar el conocimiento explícito el cual es resultado del proceso de aprendizaje de las aulas de UDIN. Siendo la retroalimentación de este a la misma comunidad académica una base para realizar una métrica y establecer indicadores que permitan controlar y proponer acciones de mejora al sistema.

\section{REFERENCIAS}

[1] Horton, W. (2000) Designing web based training Wiley Computer Publisher, New York, NY.

[2] N. Scagnoli, "El aula virtual: usos y elementos que la componen", University Of Illinois Urbana-Champaign, 2000.

[3] Suárez Conde L. "Diseño de un aula virtual empleando la plataforma Moodle como soporte tecnológico para apoyar el trabajo colaborativo y el trabajo autónomo de los estudiantes, en el espacio académico de práctica social del plan de estudios de licenciatura en química de la Universidad Distrital Francisco José de Caldas”, Universidad Distrital francisco José de caldas, (29 de febrero de 2016; Pág: 29)

[4] Universidad Distrital Francisco José de Caldas. "Sistema de gestión académica (SGA)”. Extraído de: https://udistrital.edu.co/condor/

[5] Arraez, Freddy. (2020). Gestión del Conocimiento. [en línea] http://www.aprender.org.ar/aprender/artículos/conocimiento.htm

[6] Cordero Borjas, A. E., \& García Fernández, F. (2008). «Knowledge Management and Work Teams» Observatorio Laboral Revista Venezolana, 43-64.

[7] Medina García Víctor Hugo, Medina Estrada Lina María y Tarazona Bermúdez Giovanny Mauricio. (2019). "Investigación en Ingeniería apoyada por la gestión del conocimiento y la internet social", libro, Doctorado en Ingeniería - Universidad Distrital Francisco José de Caldas. Amadgraf Impresores Ltda. $1^{a}$ Ed. Bogotá, Colombia.

[8] Juan Gabriel Cegarra Navarro (Autor), Aurora Martínez Martínez. (2017). Gestión del conocimiento. Una ventaja competitiva. ESIC Editorial. Libros Profesionales de Empresa. Vol.1.

[9] Medina García Víctor Hugo, Medina Estrada Lina María and Rivas Trujillo Edwin. (2018). "Integral Knowledge Management System in Health”, in Journal Comunications in Computer and Information Science 877. Knowledge Management in Organizations, Ed. SpringerVerlag Berlin.

[10] Oficina Asesora de Planeación y Control. "Gestión de los sistemas de información y las telecomunicaciones”. Universidad Distrital Francisco José de Caldas. Extraído de: http://planeacion.udistrital.edu.co:8080/sigud/pa/gsit

[11] Ortega Carrillo José Antonio. (2011). La investigación educativa como vía para la profesionalización de la docencia. Fundación Dialnet, Vol. 2, No. 3 págs. 1-16

[12] Rosenberg, M.J. (2001) E-Learning: Strategies for Delivering Knowledge in the Digital Age. McGraw-Hill, New York.

[13] Nonaka, I., \& Takeuchi, H. (1995). The knowledge creating company. New York: Oxford University Press.

$19^{\text {th }}$ LACCEI International Multi-Conference for Engineering, Education, and Technology: "Prospective and trends in technology and skills for sustainable social development" "Leveraging emerging technologies to construct the future", Buenos Aires -Argentina, July 21-23, 2021. 